\title{
Food Fraud Kongress: Interaktiver Fachaustausch zur Bekämpfung von Lebensmittelbetrug in Deutschland und Europa
}

\author{
Reinhard Gerlach ${ }^{1}$
}

Published online: 17 August 2017

(c) The Author(s) 2017. Dieser Artikel ist auf Springerlink.com mit Open Access verfügbar.

Zusammenfassung „Food Fraud“ war das Motto der gemeinsamen Veranstaltung des Bundesamts für Verbraucherschutz und Lebensmittelsicherheit (BVL) und des Joint Research Centers (JRC) der Europäischen Kommission am 12. und 13. Juni 2017 in Berlin. Im Zentrum des Kongresses stand die Revision der EU-Kontrollverordnung, die im Frühjahr 2017 in Kraft getreten ist. Diese neue rechtliche Ausgangssituation bietet den Rahmen, um sich mit den Auswirkungen der revidierten EU-Kontrollverordnung auf das Thema Food Fraud zu befassen. Die Veranstaltung richtete sich an betroffene Akteure und Stakeholder und bot eine gute Basis für einen regen Erkenntnis- und Entwicklungsaustausch. Neben den Vorträgen wurde außerdem ein „World Café“ organisiert, um gemeinsam mit den Teilnehmern die Anforderungen an die Lebensmittelüberwachung, die Analytik und die Wirtschaft, im Bereich der Bekämpfung von Food Fraud interaktiv zu beleuchten und so Strömungen, Entwicklungspotenziale und Anregungen $\mathrm{zu}$ bündeln und erkenntnisorientiert zu diskutieren. Es sollten somit auch wichtige Ansätze zur Umsetzung der neuen Kontrollverordnung erarbeitet werden.

Abstract „Food Fraud“ was the motto of the joint congress organized by The German Federal Office for Consumer Protection and Food Safety (BVL) and the

Reinhard Gerlach

104@bvl.bund.de

1 Bundesamt für Verbraucherschutz und Lebensmittelsicherheit (BVL), Referat 104:

Krisenmanagement, Schnellwarnsysteme, Mauerstrasse 39-42, 10117 Berlin, Deutschland
Joint Research Center of the European Commission (JRC) from 12th to 13th of June 2017 in Berlin. In addition to a number of carefully selected presentations, the organizers hosted a "World Café" on the second day of the congress. The event illuminated the requirements for food monitoring, analysis and the economy in the area of combating food fraud in a versatile way, thus stimulating suggestions, development potentials and currents from the different participants' circles and to discuss them in an epistemological manner. Important approaches to the implementation of the new Official Control Regulation (OCR) should therefore be drawn up. The possibility of active participation as well as the rotation of the groups of participants between the topics was a successful change from the series of presentations, which are usually dominating such events, followed by a linear discussion.

\section{Einleitung}

Der Kongress "Food Fraud" wurde vom Bundesamt für Verbraucherschutz und Lebensmittelsicherheit (BVL) in Zusammenarbeit mit der Gemeinsamen Forschungsstelle der Europäischen Kommission (JRC) organisiert. Das JRC unterstützt als wissenschaftliche Organisation der Europäischen Kommission die europäische Politik durch unabhängige wissenschaftsbasierte Beratung. Annähernd 180 Teilnehmer aus Wirtschaft, Wissenschaft und verschiedenen Behörden diskutierten, wie sich Lebensmittelüberwachung auf die Arbeit der jeweiligen Institutionen auswirkt, welche Rolle die Wirtschaft einnimmt und 
welche Anforderungen sich daraus im Bereich der Analytik ergeben.

\section{Inhalte}

\subsection{Vorträge}

Die Prävention von Lebensmittelbetrug obliegt in erster Linie den Lebensmittelunternehmen selbst. Diese Kernaussage untermauerte der Jurist und Rechtswissenschaftler Professor Alfred Hagen Meyer mit der Feststellung, dass die jeweiligen Qualitätsmanagementsysteme oftmals nicht ausreichten, um von Unternehmensseite effektiv gegen Lebensmittelbetrug vorzugehen. In seinen Ausführungen betonte er, dass Lebensmittelbetrug und Profitgier bei den Tätern unmittelbar zusammengehörten und diese Profite entweder durch eine starke Preisdifferenz oder schlicht durch die Masse an produzierten und verkauften Produkten erzielt würden. Das größte Potenzial für präventive Maßnahmen gegen Lebensmittelbetrug sieht Meyer vorrangig bei den herstellenden und verarbeitenden Unternehmen, da diese als Anfangsglied der Lebensmittelkette bereits grundlegende Bereiche in der Lebensmittelerzeugung abdeckten. Hieraus leitet er den klaren Auftrag für die Überwachungsbehörden ab, die mit ihren Überwachungstätigkeiten nach den Vorgaben der europäischen Kontrollverordnung (EU) 2017/625 auf betrügerische Praktiken mittels regelmäßigen, unangekündigten und risikobasierten Kontrollen reagieren müssten.

Die Bekämpfung von Lebensmittelbetrug als komplexe Herausforderung wurde von Dr. Gerd Fricke vom BVL in den Mittelpunkt seiner Ausführungen gestellt. In einer Welt globaler Warenströme und hochvernetzter Warenketten habe das betrügerische Vorgehen bei der Manipulation von Lebensmitteln heute eine Dimension und Komplexität erreicht, der nur über die koordinierte staaten- und behördenübergreifende Netzwerkarbeit adäquat begegnet werden könne, so Fricke. Den Dreh- und Angelpunkt dieser Netzwerkarbeit bildet das europäische Food Fraud Network (FFN), in dem sich das BVL seit 2013 als nationale Kontaktstelle u.a. um den koordinierten Austausch mit Behörden und Stakeholdern im In- und Ausland kümmert.

Dr. Gudrun Gallhoff von der Generaldirektion Gesundheit und Lebensmittelsicherheit der Europäischen Kommission (DG SANTE) stellte insbesondere die gute und intensive Zusammenarbeit der Behörden auf nationaler und internationaler
Ebene in den Fokus und betonte die Wichtigkeit des nahtlosen Ineinandergreifens von Kontrolle und Strafverfolgung zur effektiven Bekämpfung von betrügerischen Praktiken in der Lebensmittelproduktion. Die EU nehme hierbei besonders im internationalen Kontext eine entscheidende koordinierende Rolle ein und bilde durch Bereitstellung verschiedener IT-Ressourcen und -Systeme, aber auch durch die Vorgabe von EU-weiten Kontrollplänen und gemeinsamen Trainingsmöglichkeiten für Kontrolleure, die Grundlage für ein erfolgreiches Vorgehen gegen Lebensmittelmanipulation und -betrug.

Nach Auffassung von Andy Morling, Chef der „National Food Crime Unit“ der britischen Food Standards Agency (FSA), sollte man bei der Betrachtung des Deliktsbereichs stets von „Food Crime“, also von Lebensmittelkriminalität sprechen, um die Tragweite und das große Gefahrenpotenzial deutlich hervorzuheben sowie die Zusammenarbeit mit den Strafverfolgungsbehörden zu erleichtern. Gerade der Umstand, dass es den Fälschern darauf ankommt, möglichst lange und möglichst unerkannt zu agieren, zeige die hohe Wahrscheinlichkeit, dass ein Großteil der Konsumenten auf die eine oder andere Weise unbemerkt bereits Opfer von Lebensmittelbetrug bzw. -kriminalität wurde. Demnach formulierte Morling auch sehr treffend: „But absence of evidence is not evidence of absence“"

Die Aktivitäten zur Bekämpfung von Lebensmittelbetrug in Bayern wurden von Dr. Peter Wallner, Leiter der Spezialeinheit Lebensmittelsicherheit beim Bayrischen Landesamt für Gesundheit und Lebensmittelsicherheit (LGL), erläutert. Besonders die Entwicklung und Anwendung eines Frühwarnsystems zum Erkennen bestimmter Markttendenzen, welche sich wiederum begünstigend auf die Lebensmittelkriminalität auswirken könnten, stieß auf großes Interesse und zeige eindrücklich die Wichtigkeit, bereits im frühestmöglichen Produktionsprozess die Umstände in der Lebensmittelproduktion zu beobachten, um mögliche Gefahrenpotenziale richtig einschätzen und bewerten zu können.

Dass strafrechtliche Sanktionen ein wichtiger Bestandteil der Bekämpfung von Lebensmittelbetrug sein sollten, erläuterten die Staatsanwälte Frau Dr. Anja Wüst und Dr. Wanja Welke. Bevor es zur Anwendung von strafrechtlichen Instrumenten kommen kann, obliegt es den Lebensmittelüberwachungsbehörden, durch die Abgabe des Falles an die Staatsanwaltschaft die Einleitung eines etwaigen Strafverfahrens zu ermöglichen. Folglich kommt es bereits zum Zeitpunkt der Kontrolle maßgeblich auf 
die Beweissicherstellung an, da in dieser Phase entscheidende Weichen gestellt werden, ob der Straftatbestand des Betrugs gemäß § 263 Strafgesetzbuch (StGB) erfüllt ist oder aber lebensmittelrechtliche Vorschriften ( $\S 58$ und 59 Lebensmittel- und Futtermittelgesetzbuch (LFGB)) einschlägig sind, die im Bereich der Ordnungswidrigkeiten liegen. Eine Verurteilung wegen Betruges setzt die Feststellung eines Vermögensschadens beim Geschädigten voraus. Des Weiteren muss in diesem Zusammenhang der Vorsatz nachgewiesen werden. Laut der beiden Staatsanwälte sind diese Feststellungen in der Praxis nicht ohne weiteres mit ausreichender Bestimmtheit zu treffen. Dementsprechend positiv wurde von ihnen bemerkt, dass Lebensmittelbetrug durch die geänderte Kontrollverordnung jetzt weiter in den Fokus rücke.

Annegret Falter forderte als Vorsitzende des Vereins Whistleblower-Netzwerk e.V. ein Gesetz zum Schutz für Hinweisgeber, da diese unter der derzeitigen Rechtsprechung über keinen ausreichenden Schutz im Hinblick auf ihre Identität verfügen würden. Dies sei aber vor allem aus sicht der Überwachungsbehörden von entscheidender Wichtigkeit, da meist erst über Hinweise von intern agierenden Personen wichtige Informationen in Bezug auf Manipulationen im Lebensmittelgewerbe verfügbar wären. Um die Hemmschwelle zur Informationsweitergabe aufgrund von möglichen Suppressionen aus dem Umfeld einer hinweisgebenden Person zu senken, sieht Frau Falter deutlichen Nachbesserungsbedarf seitens des Gesetzgebers.

Die parlamentarische Staatssekretärin des Bundeministeriums für Landwirtschaft und Ernährung (BMEL), Dr. Maria Flachsbarth, betonte in ihren Ausführungen die zentrale Rolle Deutschlands im gesamteuropäischen Kontext. Als Ein-, Aus- und Durchfuhrland würden enorme Warenmengen über ein hochkomplexes Netz aus Warenströmen und Lieferketten bewegt. Neben der notwendigen europäischen Lösung im Bereich der Lebensmittelkriminalität ging sie dabei intensiv auf die nationale Strategie zur Bekämpfung von Lebensmittelbetrug ein, die vom BVL umgesetzt wird, und zeichnete ein Bild der verschiedenen sich ergänzenden Elemente, welche diese Strategie stützen. Hierbei betonte sie besonders die präventiven Maßnahmen, den verbesserte Nachweis von Manipulation durch die Weiterentwicklung analytischer Methoden sowie die Stärkung der behördlichen Zusammenarbeit betont.

In Bezug auf die Analytik bekräftigte die Leiterin der Abteilung Untersuchungen beim BVL, Dr. Petra Gowik, die Notwendigkeit, vorhandene europaweite
Forschungsergebnisse zur Echtheit und Integrität von Lebensmitteln zusammenzuführen und den Beteiligten zugänglich zu machen. Ihren Ausführungen nach wird es zukünftig von entscheidender Bedeutung sein, die Vorgaben der neuen Kontrollverordnung zur Akkreditierung sowie die Einbeziehung weiterer Rechtsbereiche im Hinblick auf die Lebensmittelechtheit und -integrität der Lebensmittelkette strukturiert und koordiniert umzusetzen. Aus diesem Grund wurde kürzlich vom Bundesministerium für Ernährung und Landwirtschaft (BMEL) die Einrichtung eines nationalen Referenzzentrums für die Echtheit und Integrität von Lebensmitteln (NRZ-EIL) am Max Rubner-Institut in Kulmbach beschlossen.

In die gleiche Richtung, wenn auch auf europäischer Ebene, gingen die Erläuterungen von Dr. Gudrun Gallhoff. Sie stellte die Anforderungen an die Einrichtung und die Aufgaben eines europäischen Referenzzentrums für die Authentizität und Integrität der Lebensmittelkette vor und betonte in diesem Zusammenhang die zentrale Rolle der Europäischen Kommission bei der Unterstützung der Mitgliedstaaten. Diese basiere unter anderem auf der koordinierten Vernetzung, der Aufstellung von Kontrollplänen sowie der Aus- und Weiterbildung der Beteiligten durch spezielle Trainingsangebote.

Die Forschungsansätze auf Bundesebene am Max Rubner-Institut (MRI) wurden von Dr. Lara Frommherz vorgestellt. Als Einrichtung innerhalb des Geschäftsbereichs des BMEL werden am MRI vor allem die Belange des gesundheitlichen Verbraucherschutzes bei Ernährung und Lebensmitteln erforscht. Das MRI verfügt über insgesamt acht Institute an vier Standorten in Deutschland und ist somit nahezu optimal auf die vielfachen Anforderungen der Lebensmitteluntersuchungen eingestellt. Nicht zuletzt aufgrund dieser weitgefächerten Fachkompetenz, die es ermöglicht, die verschiedensten Lebensmittel und Rohstoffe auf sämtlichen Produktionsstufen umfassend $\mathrm{zu}$ analysieren und als Referenzmaterial bereit zu stellen, wurde das MRI mit der Einrichtung des bereits erwähnten nationalen Referenzzentrums NRZ-EIL betraut.

$\mathrm{Zu}$ den speziellen Anforderungen bei der analytischen Authentizitätsprüfung gab Dr. Carsten FauhlHassek vom Bundesinstitut für Risikobewertung (BfR) einen umfassenden Überblick. Er machte wiederholt deutlich, dass für eine genaue und effektive Bewertung der Analyseergebnisse anhand der festgelegten Parameter neben den verschiedenen Forschungs- und Analyseansätzen die zeitnahe Bereitstellung entsprechender Referenzmaterialien in ausreichender Menge 
sowie die intensive behördenübergreifende Vernetzung als große Herausforderung angesehen wird.

Eine sehr detaillierte und umfassende Darstellung der speziellen Untersuchungstechniken und -verfahren vermittelte Dr. Manfred Stoyke vom BVL und wies darauf hin, dass allein in der amtlichen Sammlung von Untersuchungsverfahren (ASU) ca. 1.500 Methoden für Lebens- und Futtermittel vorliegen, welche zum Teil auch Eingang in die DIN- und CEN-Normung gefunden haben, jedoch häufig noch an die speziellen Anforderungen im Bereich der Lebensmittelkriminalität angepasst werden müssten. Hierbei wird das BVL mittels der entsprechenden $§ 64$ LFGB-Arbeitsgruppen einen wichtigen Beitrag zur Validierung und Veröffentlichung der Methoden leisten.

Die Bekämpfung von Lebensmittelbetrug und Lebensmittelkriminalität ist in vielerlei Hinsicht ein Thema, welches Wirtschaft, Politik und Wissenschaft weiterhin beschäftigen wird. Dementsprechend mahnend fielen auch die Schlussworte des Präsidenten des BVL, Dr. Helmut Tschiersky aus. Zusammenfassend betonte er, dass zum einen der bessere Schutz für Hinweisgeber eine besondere Zukunftsaufgabe sei und zum anderen die Zusammenarbeit und Kommunikation aller Beteiligten in diesem komplexen System über den Ausbau der bereits bestehenden Netzwerke hinaus verbessert werden müsse.

\subsection{World Café}

Um dieser Vielzahl an Eindrücken aus den erkenntnisgeladenen Vorträgen mittels Diskussions- und Austauschmöglichkeit in geeigneter Weise begegnen zu können, wurde am zweiten Tag der Veranstaltung ein „World Café“ organisiert, um die Anforderungen an die Wissenschaft, die Wirtschaft und die behördliche Lebensmittelüberwachung im Bereich der Lebensmittelkriminalität vielseitig zu beleuchten. Das World Café sollte Strömungen, Entwicklungspotenziale und Anregungen aus den verschiedenen Teilnehmerkreisen bündeln, um diese erkenntnisorientiert zu diskutieren sowie wichtige Ansätze zur Umsetzung der neuen Kontrollverordnung zu erarbeiten. Das Diskussionsformat bot die Möglichkeit zur Interaktion und aktiver Meinungsäußerung. Die so gewonnen Erkenntnisse konnten dabei verdichtet und zueinander in Bezug gesetzt werden. Die aktiven Teilnehmer des World Cafés trugen Schlüsselideen oder Themen zu anderen Tischen, tauschten Perspektiven aus und bereicherten so in großem Maße den Lösungsfindungsprozess (Abb. 1).
Das World Café des Food Fraud Kongresses wurde in vier Hauptthemengebiete gegliedert:

- Anforderungen an die Analytik,

- Anforderungen an die Wirtschaft und den gegenseitigen Informationsaustausch zwischen Wirtschaft und Behörden,

- Anforderungen an die Lebensmittelüberwachung und

- Anforderungen an den Umgang mit Informationen und Hinweisen.

Zur besseren Unterscheidung wurde zudem mit einer farblichen Markierung gearbeitet, um eine klare und nachvollziehbare Gruppenwechselstruktur zu visualisieren. $\mathrm{Zu}$ Beginn des World Cafés bekam jeder Teilnehmer eine entsprechende Farbkarte überreicht und war somit einer Gruppe zugeordnet, was wiederum den Rotationsprozess deutlich vereinfachte und einen reibungslosen Wechsel der Gruppen trotz der großen Teilnehmerzahl jederzeit ermöglichte. In Abb. 2 ist schematisch die farbliche Zuordnung der Thementische in Verbindung mit dem vorgesehenen Rotationsprinzip dargestellt.

Ebenso wie die strukturelle Gliederung des gesamten World Cafés war die jeweilige Diskussion der vier Themengebiete einem geordneten Ablauf unterworfen, welcher sich in vier Phasen gliederte und von den im Vorfeld bestimmten Moderatoren begleitet und durchgesetzt wurde. In Phase eins wurde ein themeneinleitendes Brainstorming zur allgemeinen Thesenfindung mit zielgerichteter Problemansprache durchgeführt. Phase zwei war vor allem von der Validierung der im Brainstorming erarbeiteten Antworten geprägt und diente in gewisser Weiser einer Vorfilterung der wichtigsten Denkansätze. Phase drei sollte bereits ziel- und ergebnisorientiert hervorbringen, wer in welchem Zeitrahmen bestimmte Aufgaben im Hinblick auf die vertiefte Befassung mit den Auswirkungen der revidierten EU-Kontrollverordnung übernehmen könnte. Die Schlussphase bildete die Phase vier, welche wiederum zur Validierung der Ergebnisse und der Prüfung der Realisierbarkeit dienen sollte.

Zur Visualisierung und Präsentation der gesammelten Ideen bzw. Erkenntnisse aus der Gruppenarbeit wurden verschiedene Techniken angewendet. Ein Baustein war das Anbringen von Haftnotizzettel mit anschließender galerieartiger Gruppierung an einer der Raumwände. Diese Zuordnung hatte den entscheidenden Vorteil, dass zusammenhängende Ideen gut sichtbar gemacht wurden und eine Gewichtung hinsichtlich der Realisierbarkeit wesentlich vereinfachte. In Phase vier 


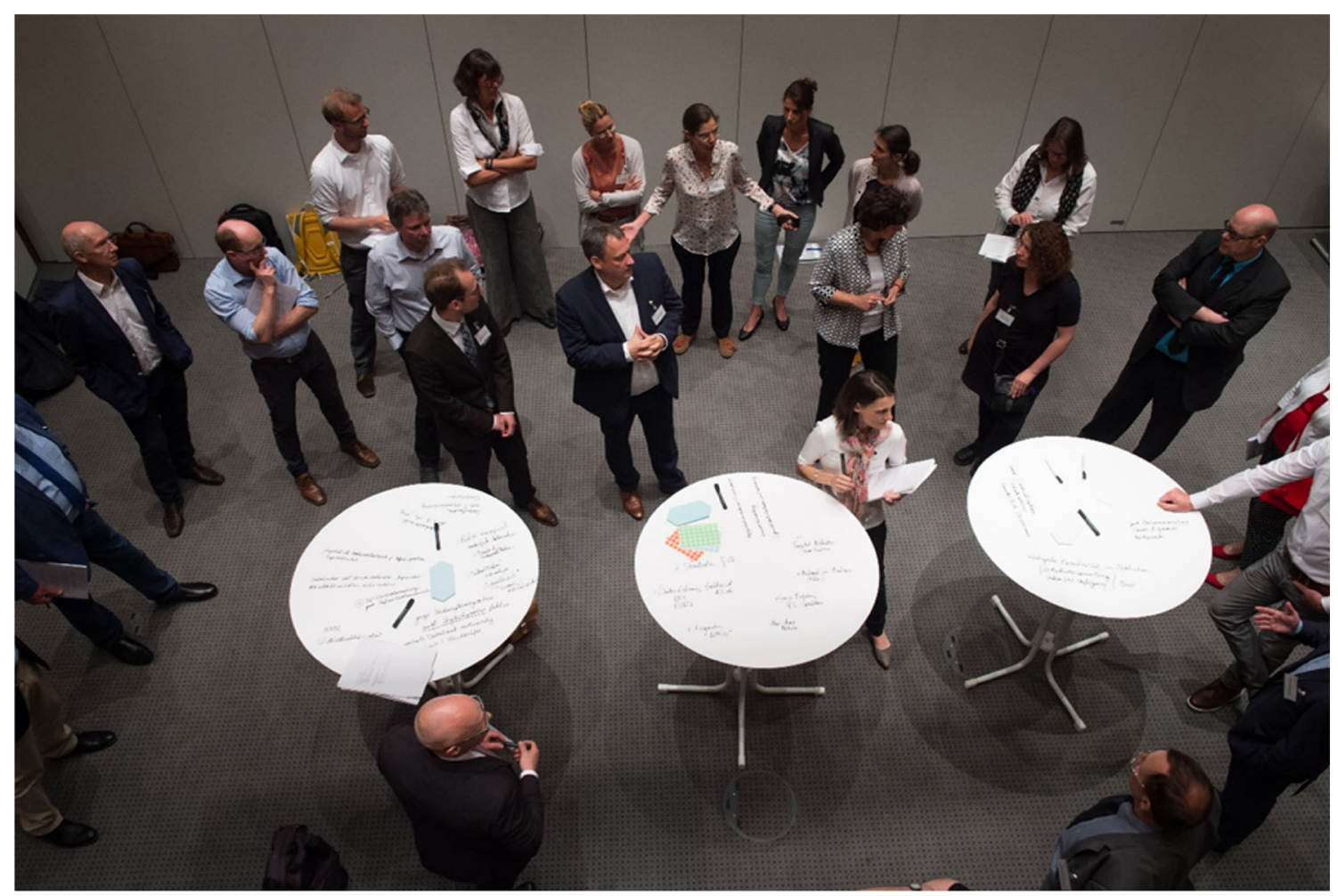

Abb. 1 Die Teilnehmer der World Cafés an den interaktiven Tischen (Foto: Pflug/BVL)

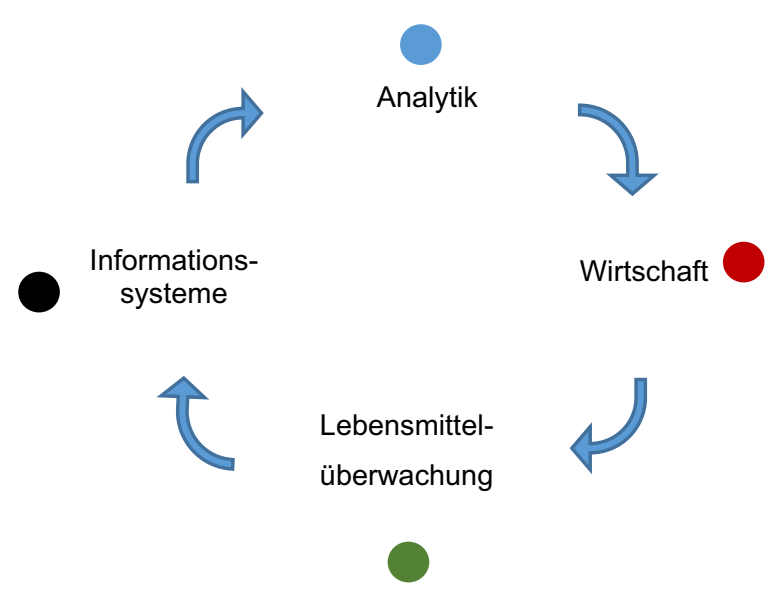

Abb. 2 Rotation und Farbzuordnung Thementische

wurde dann mittels verschiedenfarbiger Klebepunkte die bereits erwähnte Gewichtung noch einmal visuell betont (Abb. 3).

Die Ergebnisse zeigten, dass sich mittelfristig drei große Handlungsfelder ergeben. Im Zuge der Aufwertung des Deliktbereiches Lebensmittelbetrug bzw. Food Fraud hin zur verbal und inhaltlich deutlich geschärften Begrifflichkeit der Lebensmittelkriminalität bzw. Food Crime sollte es nach übereinstimmender Meinung vieler Teilnehmer entweder eine Erweiterung der polizeilichen Kompetenzen innerhalb der Lebensmittelüberwachung oder aber eine Verschiebung bestimmter Tätigkeiten in den Polizeibereich in Verbindung mit paralleler Schulung im Lebensmittelsektor geben. Gleichzeitig wäre es im nachgelagerten Ermittlungsbereich, also im Gebiet der Tatbe-standsbeurteilung von entscheidender Bedeutung, vermehrt Schwerpunktstaatsanwaltschaften zu bilden bzw. die Vermittlung von Lebensmittel-Fachkompetenz an Staatsanwälte und Richter voran zu treiben. In den Augen der meisten Teilnehmer spiegelt sich hier der Wunsch, durch erweiterte Ermittlungs- und Eingriffskompetenzen in Verbindung mit aktueller fachlicher Expertise dem Lebensmittelbetrug zeitnah begegnen zu können und die entsprechende Reaktion durch Prävention zu beschleunigen.

Das zweite große Handlungsfeld wird durch die rechtsgültige Regelung des Hinweisgeberschutzes geprägt. Hinweise aus dem Bereich des sogenannten Whistleblowings sind wertvoll und wichtig zur Bekämpfung von Lebensmittelmanipulation und -betrug. In vielen Fällen sind die Hinweise von internen Mitarbeitern die einzige Möglichkeit für Behörden, etwaigen Regelverletzungen auf die Spur zu kommen. Für ein effektives Hinweisgebersystem müssen allerdings der Arbeitnehmer- sowie der 


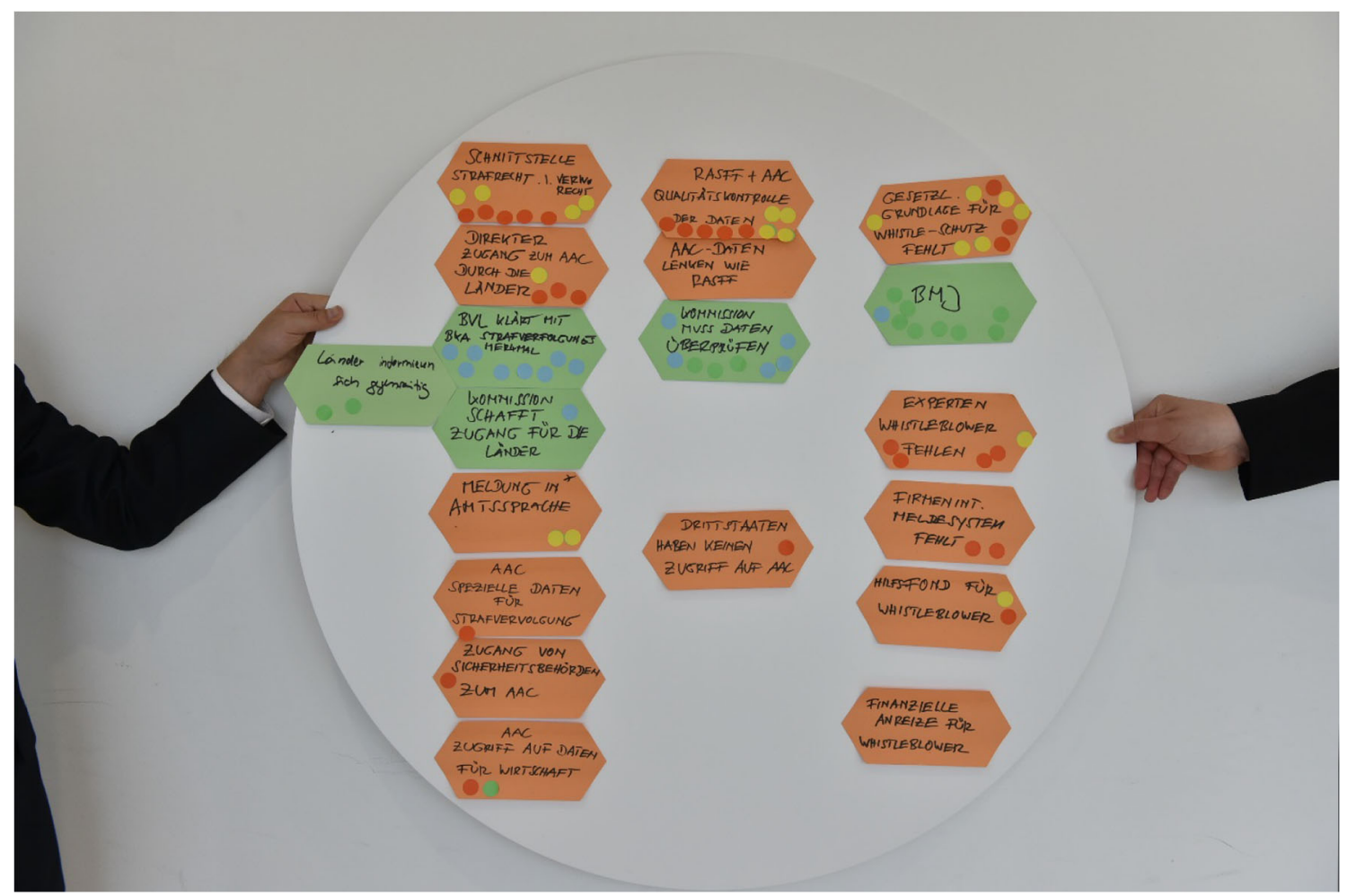

Abb. 3 Die gesammelten Ideen bzw. Erkenntnisse des World Cafés (Foto: Pflug/BVL)

Daten- und sogar Identitätsschutz gewährleistet sein, um die Whistleblower vor etwaigen Suppressionen aus dem näheren Umfeld zu schützen. Dies muss durch entsprechende gesetzliche Regelungen auf den Weg gebracht werden. Äußerst positiv wurde in diesem Zusammenhang die Äußerung der Parlamentarischen Staatssekretärin Flachsbarth aufgenommen, die sich für eine entsprechende Prüfung eines solchen Gesetzgebungsverfahrens aussprach.

Der nach Meinung vieler Teilnehmer dringlichste Verbesserungsbedarf und damit das dritte große Handlungsfeld wurde in der Verbesserung der Zusammenarbeit und Kommunikation von Wirtschaft und Behörden gesehen. Zukünftig wird es von entscheidender Bedeutung sein, eine gemeinsame Strategie zu entwickeln und zu etablieren, bei der es zum einen um die Erstellung und Einführung von Konzepten zur Gefahrenanalyse durch Implementierung von Standards und Normen geht und zum anderen um den intensiven Informationsaustausch zu bestimmten Produkten, Gefahren und Herkunfts- bzw. Transitländern im Sinne eines Frühwarnsystems.

\section{Fazit}

Das „World Café“ trug im Wesentlichen dazu bei, dass sich die Teilnehmer des Kongresses in wichtigen Punkten zum Thema Food Fraud aktiv mit ihren Ideen und Erfahrungen einbringen konnten und bei der Problemerörterung in sehr direkter Weise beteiligt waren. Gerade diese Art der unmittelbaren Kommunikation auf gleicher Ebene kann Denkansätze und Probleme der unterschiedlichen Positionen aus Wirtschaft, Wissenschaft und behördlicher Verwaltung einander näherbringen und im Idealfall gemeinsame Strategien ausbilden, um in der Konsequenz dem äußerst vielfältigen Phänomen des Lebensmittelbetruges Einhalt gebieten zu können.

Die Möglichkeit der aktiven Mitgestaltung sowie die Rotation der Teilnehmergruppen zwischen den Thementischen war eine gelungene Abwechslung zu den sonst bei derartigen Veranstaltungen dominierenden Vortragsreihen mit anschließender linearer Diskussion und trug im besonderen Maße zu einer offenen, von Aktivität geprägten Stimmung bei, die von vielen Teilnehmern als äußerst positiv empfunden wurde. 
Im Ergebnis zeigten sich im Grunde drei Handlungsfelder, welche nach Ansicht der meisten Teilnehmer besonders dringlich waren:

- Die Erweiterung der polizeilichen Kompetenz bei der Lebensmittelüberwachung inklusive des Einrichtens weiterer Schwerpunktstaatsanwaltschaften. Dazu gehört die bessere Verzahnung von behördlichen Kontrollen und Kompetenzen sowie eine intensivere juristische Bewertung.

- Ein verbesserter Schutz so genannter Whistleblower inklusive der entsprechenden Daten bzw. Identitäten.
- Eine verbesserte Kommunikation und Zusammenarbeit von Wirtschaft und Behörden.

Open Access This article is distributed under the terms of the Creative Commons Attribution 4.0 International License (http://creativecommons.org/licenses/by/4.0/), which permits unrestricted use, distribution, and reproduction in any medium, provided you give appropriate credit to the original author(s) and the source, provide a link to the Creative Commons license, and indicate if changes were made. 